JULIA TAMER LANGEN

\title{
A Lei Modelo da UNCITRAL e a disciplina da insolvência transnacional no Brasil
}

UNIVERSIDADE DE SÃO PAULO

FACULDADE DE DIREITO

São Paulo - SP

2020 
JULIA TAMER LANGEN

\section{A Lei Modelo da UNCITRAL e a disciplina da insolvência transnacional no Brasil}

Dissertação de Mestrado apresentada para a Banca Examinadora do Programa de Pós-Graduação em Direito, da Faculdade de Direito da Universidade de São Paulo, na área de concentração Direito Comercial, sob a orientação do Professor Dr. Paulo Fernando Campos Salles de Toledo

UNIVERSIDADE DE SÃO PAULO

FACULDADE DE DIREITO

São Paulo - SP

2020 
Catalogação da Publicação

Serviço de Biblioteca e Documentação

Faculdade de Direito da Universidade de São Paulo

Langen, Julia Tamer

A Lei Modelo da UNCITRAL e a disciplina da

insolvência transnacional no Brasil ; Julia Tamer

Langen ; orientador Paulo Fernando Campos Salles de

Toledo -- São Paulo, 2020.

142

Tese (Doutorado - Programa de Pós-Graduação em

Direito Comercial) - Faculdade de Direito,

Universidade de São Paulo, 2020.

1. Insolvência Transnacional. 2. Lei Modelo da

UNCITRAL. 3. Direito Privado. 4. Direito Falimentar.

I. Toledo, Paulo Fernando Campos Salles de, orient.

II. Título. 


\section{Banca examinadora:}

1. Orientador: Paulo Fernando Campos Salles de Toledo

2. Examinador(a):

3. Examinador(a):

4. Examinador(a): 


\title{
RESUMO
}

\section{Julia Tamer Langen. A Lei Modelo da UNCITRAL e a Disciplina da Insolvência}

Transnacional no Brasil. 2020. 142 f. Dissertação (Mestrado em Direito) - Departamento de Direito Comercial, Faculdade de Direito, Universidade de São Paulo, São Paulo.

\begin{abstract}
A Lei n ${ }^{\circ} 11.101 / 2005$ não disciplina insolvências transnacionais, a despeito da proliferação de grupos societários transnacionais e do fenômeno da globalização. Diante da perspectiva de adoção pelo Brasil de regras inspiradas no universalismo modificado e na Lei Modelo da Comissão das Nações Unidas para o Direito Comercial Internacional (UNCITRAL), o escopo deste trabalho é um estudo dogmático do direito da insolvência transnacional, a partir da Lei Modelo, de princípios gerais informadores da matéria, e da experiência norteamericana no tema. Além disso, o trabalho analisa a compatibilidade e os benefícios do direito projetado com o ordenamento jurídico brasileiro atualmente em vigor concluindo que a adoção da Lei Modelo seria possível e bem-vinda. A partir desse estudo, pretendese contribuir, sobretudo, com a interpretação e a aplicação consistentes e apropriadas do direito projetado na matéria.
\end{abstract}

Palavras-chaves: Insolvência transnacional - Lei Modelo da UNCITRAL sobre insolvência - Universalismo Modificado - PL n 6.229/2005. 


\begin{abstract}
Julia Tamer Langen. The UNCITRAL Model Law and the discipline of transnational insolvency in Brazil. 2020. 142 p. Paper (Master of Laws) - Department of Commercial Law, Faculty of Law, University of São Paulo, São Paulo.
\end{abstract}

Law n. 11,101 / 2005 does not regulate cross-border insolvencies, despite the proliferation of transnational corporate groups and the phenomenon of globalization. Given the prospect of Brazil adopting rules inspired by modified universalism and United Nations Committee on International Trade Law (UNCITRAL) Model Law, the scope of this study is a dogmatic study of cross-border insolvency law, in light of the UNCITRAL Model Law, principles, and the US experience. In addition, the study analyzes the compatibility and benefits of the projected law with the current Brazilian legal system and concludes that adoption of the Model Law would be possible and welcomed. The study is intended to contribute, above all, with consistent and appropriate interpretation and application of the projected law in the matter.

Key-words: Cross-border Insolvency - UNCITRAL Model Law on Insolvency - Modified Universalism - Bill of Law n. 6,229/05. 


\section{Lista de Abreviaturas e Siglas}

CF Constituição da República Federativa do Brasil de 1988

COMI Centro de interesses principais do devedor (a expressão em inglês centre of main interests ou na forma abreviada COMI, que é amplamente utilizada)

CPC/15 Código de Processo Civil brasileiro (Lei n ${ }^{\circ}$ 13.105/2015)

CPC/73 Código de Processo Civil brasileiro revogado (Lei ${ }^{\circ}$ $5.869 / 1973)$

CPC/39 Código de Processo Civil brasileiro revogado de 1939 (Decreto-Lei no 1.608/1939)

Des. Desembargador

DJe Diário Judicial Eletrônico

Grupo de Trabalho Grupo de Trabalho instituído com a finalidade de estudar, consolidar e propor medidas voltadas ao aprimoramento da Lei $\mathrm{n}^{\circ} 11.101$, de 9 de fevereiro de 2005, e de outros instrumentos legais associados aos temas recuperação e falência de empresas, composto por Thomas Felsberg, Francisco Satiro de Souza Jr., Paulo Fernando Campos Salles de Toledo, Sheila Neder Cerezetti, Cássio Cavalli, entre outros, nos termos da Portaria $\mathrm{n}^{\circ} 467$, de 16 de dezembro de 2016 do Ministério da Fazenda

Guia de

Interpretação

Guia para a promulgação e interpretação da UNCITRAL de 2013 
Guia de Práticas

Guia Legislativo

\section{INSOL}

InsO

j.

Lei Modelo

LINDB

LRE

MIICA

PL
Guia de práticas da UNCITRAL sobre cooperação em insolvência transnacional de 2009

Guia legislativo da UNCITRAL sobre insolvência transnacional - parte um e parte dois (2004); Guia legislativo da UNCITRAL sobre o tratamento de grupos de empresas em situação de insolvência - terceira parte (2010) e Guia legislativo da UNCITRAL sobre as obrigações de diretores no período que antecede a insolvência - quarta parte (2013)

Associação Internacional denominada International Bar Association

Associação Internacional denominada Insol - International Association of Restructuring, Insolvency \& Bankruptcy Professionals

Legislação alemã sobre insolvência (InsolvezOrdnung)

Julgamento

Lei Modelo da UNCITRAL sobre insolvências transnacionais de 1997

Lei de Introdução às normas do Direito brasileiro (Dec-Lei 4.657/42)

Lei $\mathrm{n}^{\circ} 11.101 / 2005$

Model International Insolvency Code proposto pela IBA.

Projeto de Lei 
$\mathrm{PL} \mathrm{n}^{\circ}$ 6.229/05 Projeto de Lei $\mathrm{n}^{\circ}$ 6.229/2005

PL n $^{\circ} 10.220 / 18 \quad$ Projeto de Lei $n^{\circ} 10.220 / 2018$

RIE Regulamento (UE) 2015/848 do Parlamento Europeu e do Conselho da União Europeia de 20 de maio de 2015 relativo aos processos de insolvência (reformulado)

RIE 2000 Regulamento (UE) 1346/2000 do Parlamento Europeu e do Conselho da União Europeia de 29 de maio de 2000 relativo aos processos de insolvência.

REsp Recurso Especial

STF Supremo Tribunal Federal

STJ Superior Tribunal de Justiça

Substitutivo Substitutivo apresentado ao plenário da Câmara dos Deputados em 27 de novembro de 2019 relacionado ao Projeto de Lei $n^{\circ} 6.229 / 2005$, elaborado a partir do PL $\mathrm{n}^{\circ} 10.220 / 2018$

TJSP Tribunal de Justiça do Estado de São Paulo

TJRJ Tribunal de Justiça do Estado do Rio de Janeiro

UNCITRAL Comissão das Nações Unidas para o Direito Comercial Internacional (United Nations Committee on International Trade Law)

InsO Legislação alemã sobre insolvência (InsolvezOrdnung) 


\section{SUMÁRIO}

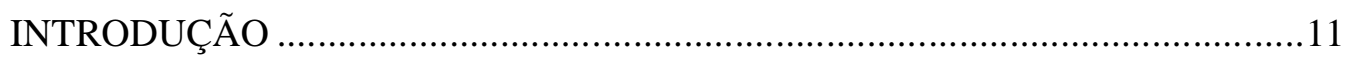

1. ALGUMAS NOTAS SOBRE A EVOLUÇÃO DAS NORMAS SOBRE INSOLVÊNCIA TRANSNACIONAL ........................................................16

1.1 Histórico dos principais sistemas normativos internacionais sobre

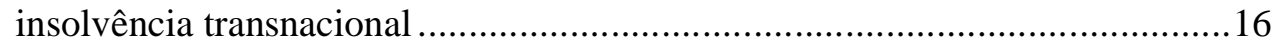

1.1.1 As origens da Lei Modelo da UNCITRAL ….................................17

1.1.2 As origens do Regulamento Europeu..........................................18

$1.2 \quad$ Histórico normativo da matéria no Brasil ........................................19

1.3 Histórico jurisprudencial sobre reconhecimento de sentença estrangeira em processos de insolvência ..........................................................................26

2. PRINCÍPIOS DE INSOLVÊNCIA TRANSNACIONAL E OS MODELOS TEÓRICOS QUE PERMEIAM A MATÉRIA...................................................30

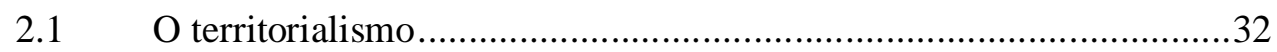

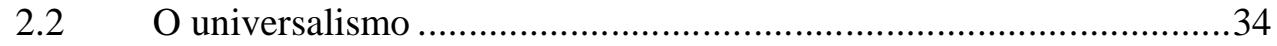

2.3 Os modelos teóricos intermediários ...............................................36

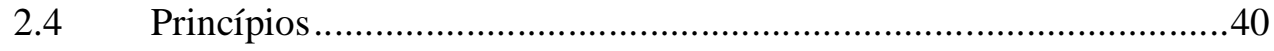

2.4.1 Princípios relacionados à jurisdição................................................40

2.4.2 Princípios processuais .............................................................41

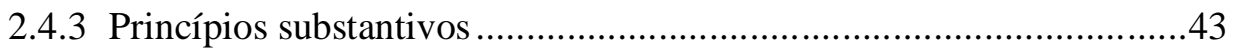

2.4.4 Relevância para o estudo de normas transnacionais de insolvência ....48

3. A LEI MODELO DA UNCITRAL SOBRE INSOLVÊNCIA

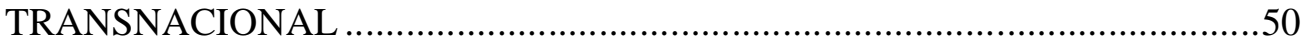

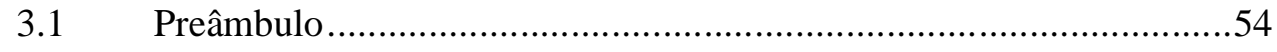

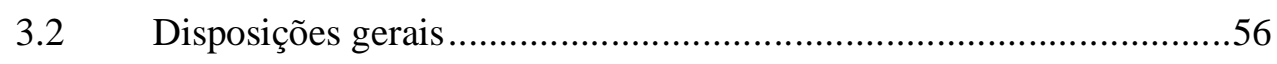

3.3 Acesso de representantes estrangeiros e credores às autoridades do país

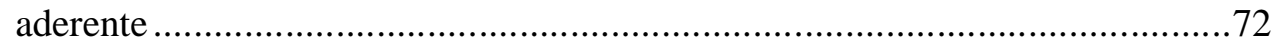

3.4 Reconhecimento de processos estrangeiros...................................76

3.5 Cooperação com autoridades e representantes estrangeiros.................79

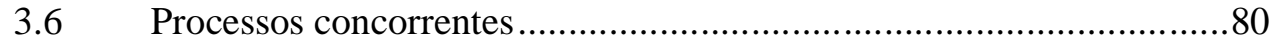

4. OUTRAS FONTES A RESPEITO DA MATÉRIA ................................83 
4.1 As recomendações do Banco Mundial ............................................... 83

4.2 O Chapter 15 do Bankruptcy Code Norte Americano ........................8 85

$4.3 \quad$ O Regulamento Europeu ...................................................... 88

4.4 Outras fontes, guias de melhores práticas, protocolos, acordos de cooperação e recomendações internacionais..................................................94

5. A CONSTRUÇÃO DO DIREITO DA INSOLVÊNCIA TRANSNACIONAL

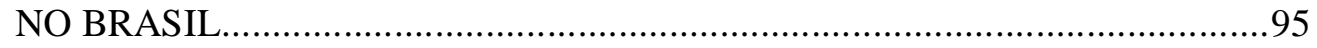

5.1 A experiência jurisprudencial brasileira em matéria de insolvência

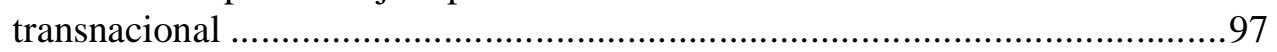

5.2 O direito projetado sobre insolvência transnacional no Brasil .......... 105

5.3 Diálogo do direito projetado com os instrumentos de cooperação jurídica

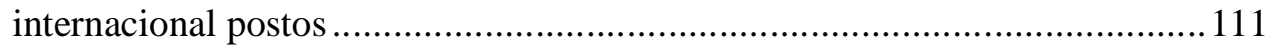

5.4 A adoção da Lei Modelo sobre insolvência transnacional pelo Brasil116

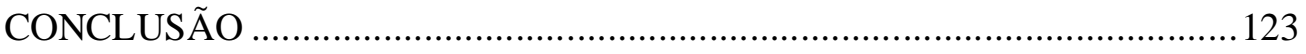

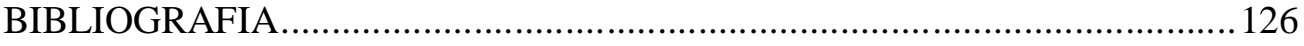




\section{INTRODUÇÃO}

Esta dissertação trata do desenvolvimento do direito positivo brasileiro sobre situações de insolvência transnacional, notadamente da perspectiva de adoção da Lei Modelo da UNCITRAL ${ }^{1}$ sobre insolvências transnacionais pelo Brasil.

Além de relevante, o tema é atual. Nas últimas décadas, a globalização impulsionou o desenvolvimento de grupos empresariais transnacionais, com ativos, dívidas e operações em diversos países. Muitas empresas brasileiras se desenvolveram para além das nossas fronteiras, constituindo subsidiárias em países estrangeiros para exercer suas atividades, captar recursos e acessar novos mercados. Da mesma forma, o Brasil recebeu investimentos de incontáveis grupos estrangeiros, que atuam no Brasil por suas subsidiárias.

Mas sociedade brasileira experimentou período de crise econômica tida por muitos analistas como a pior das últimas três décadas, que se refletiu também no aumento no número de pedidos de recuperação judicial ${ }^{2}$ e na fragilidade dos indicadores econômicos do país.

A desaceleração da economia brasileira, agravada pela queda no valor de commodities, escassez de crédito e pelos recentes escândalos de corrupção, foram determinantes para as dificuldades financeira experimentadas por relevantes grupos econômicos com operações e ativos no Brasil e no exterior, incluindo os grupos OGX, OAS, Abengoa, Schahin, Sete Brasil, Oi e Odebrecht.

A crise desses grandes grupos empresariais testou a capacidade do direito da insolvência brasileiro para enfrentar as novas situações de insolvência transnacional e evidenciou que o desenvolvimento das normas falimentares brasileiras não acompanhou a evolução dos grupos societários transnacionais nem os movimentos internacionais de uniformização de normas empresariais no contexto da globalização.

\footnotetext{
${ }^{1}$ A Lei Modelo foi elaborada em 1997 pela UNCITRAL como instrumento de soft law e contém recomendações legislativas que podem ser adotadas total ou parcialmente pelos países, como forma de harmonizar diferentes sistemas e promover a cooperação entre países em matéria de insolvência transnancional.

2 Cf. SERASA EXPERIAN. Sítio eletrônico. Disponível em: <https://www.serasaexperian.com.br/release/indicadores/falencias_concordatas.htm>. Acesso em: 2 jan. 2020.
} 
A LRE é silente quanto a insolvências transnacionais. A lacuna legislativa impôs que o Poder Judiciário encontrasse soluções para os casos submetidos à sua apreciação, contribuindo para moldar, dentro dos limites da atuação jurisdicional, algumas relevantes regras de insolvência transnacional.

Porém, a complexidade dos casos de insolvência transnacional, - que, ultima ratio, envolvem a própria soberania nacional, construída a partir de regras cogentes de delimitação do poder jurisdicional nacional e de cooperação entre jurisdições, - e a busca por segurança jurídica, evidenciam a necessidade de disciplinar a matéria.

Assim, se as grandes crises econômicas ajudam a moldar o direito falimentar ${ }^{3}$, o momento histórico é oportuno para a discussão sobre a adoção pelo Brasil da Lei Modelo, e o desenvolvimento de normas brasileiras a respeito de insolvência transnacional.

A Lei Modelo foi criada com o escopo de exemplificar as melhores práticas em matéria de insolvência transnacional, conferindo instrumentos para o acesso, a comunicação e a cooperação entre autoridades de diferentes nacionalidades. Trata-se de instrumento de soft law que contém que contém recomendação legislativa que pode ser incorporada total ou parcialmente aos ordenamentos jurídicos dos Estados pela edição de leis locais ${ }^{4}$.

No que tange às discussões acadêmicas sobre insolvência transnacional, há dois principais modelos teóricos antagônicos: o territorialismo e o universalismo. O territorialismo ignora os aspectos transnacionais da insolvência em homenagem à soberania estatal. De acordo com esse modelo teórico, o Estado tem jurisdição exclusiva sobre os bens do devedor localizados dentro de suas fronteiras. Por outro lado, o universalismo autoriza o Estado, no qual o devedor tenha estabelecido seu $\mathrm{COMI}^{5}$, a administrar o respectivo processo de insolvência ${ }^{6}$, que poderá ter reflexos em outros países a partir da abertura de processos não-princpais.

\footnotetext{
${ }^{3}$ Cf. CAMPANA FILHO, Paulo Fernando. A recuperação judicial de grupos societários multinacionais: contribuições para o desenvolvimento de um sistema jurídico brasileiro a partir do direito comparado. Tese (Doutorado) - Universidade de São Paulo, Faculdade de Direito, 241 folhas, 2013, p. 13.

${ }^{4}$ CAMPANA FILHO, Paulo Fernando. A recuperação..., p. 97-98.

${ }^{5}$ A expressão em inglês centre of main interests ou na forma abreviada COMI, que é amplamente utilizada. Examinaremos o conceito de COMI no capítulo 3.

${ }^{6}$ Cf. SOUZA JÚNIOR, Francisco Satiro; CAMPANA FILHO, Paulo Fernando. A insolvência transnacional: para além da regulação estatal e na direção dos acordos de cooperação. In: TOLEDO, Paulo Fernando
} 
A forma pura desses modelos teóricos antagônicos foi superada a partir de soluções intermediárias propostas no meio acadêmico, tais como o universalismo modificado de Jay Lawrence Westbrook ${ }^{7}$, o territorialismo cooperativo de Lynn LoPucki ${ }^{8}$ ou o universalismo coordenado de Bob Wessels 9 .

Em linha com as correntes teóricas intermediárias, a Lei Modelo prevê, por exemplo, instrumentos para cooperação e solução de conflitos de jurisdição entre Estados, medidas de combate a fraudes internacionais e lavagem de dinheiro, conferindo maior segurança jurídica para o comércio e investimentos internacionais.

O Brasil já usufruiu da cooperação de juízos estrangeiros em diversos casos de insolvência de grupos brasileiros transnacionais, como os processos de recuperação judicial da Centrais Elétricas do Pará S.A. (CELPA), do grupo Varig, do Frigorífico Independência S.A., da Rede Energia S.A. e do Grupo OAS. Nesses casos, os magistrados brasileiros contaram com a cooperação de autoridades estrangeiras a partir de instrumentos previstos na Lei Modelo e outros análogos (i.e., a revogada Section 304 do Bankruptcy Code dos Estados Unidos, sucedida pelo atual Chapter 15 do Bankruptcy Code dos Estados Unidos).

No entanto, o ordenamento brasileiro não contém normas que possibilitem à Justiça Brasileira retribuir esse tipo de iniciativa. As leis brasileiras preveem a necessidade de homologação de sentenças estrangeiras e de cooperação por meio de cartas rogatórias. Essas soluções, contudo, não atendem ao dinamismo e à necessidade de soluções céleres, que é característica dos processos de insolvência.

Os precedentes ilustram a importância do tema e o desenvolvimento que a questão teve nas últimas décadas, mas apontam a necessidade de desenvolvimento do sistema jurídico brasileiro no que toca a disciplina de direito positivo da insolvência transnacional, inclusive por meio da incorporação das recomendações da Lei Modelo.

Campos Salles de; SOUZA JÚNIOR, Francisco Satiro (coords.). Direito das Empresas em Crise: Problemas e Soluções. São Paulo: Quartier Latin, 2012, p. 123.

${ }^{7}$ Cf. WESTBROOK, Jay Lawrence. Chapter 15 at Last. American Bankruptcy Law Journal, v. 79, 2005, pp. 713-728.

${ }^{8}$ Cf. LOPUCKI, Lynn. The Case for Cooperative Territoriality in International Bankruptcy. Michigan Law Review, Vol. 98, 2000, p. 2216.

${ }^{9}$ Cf. WESSELS, Bob. EU Insolvency Regulation and Its Impact on European Business. CESifo DICE Report, v. 4, pp. 16-21, 2006. 
Assim, esta dissertação analisará conveniência de adoção, pelo legislador brasileiro de normas propostas pela Lei Modelo em linha com o universalismo modificado, como forma de incremento segurança jurídica e a preservação de valor dos ativos para aumentar o bem-estar social.

A eleição da Lei Modelo como arquétipo normativo não é devida apenas ao fato de que a coordenação internacional de processos de insolvência demanda, o tanto quanto possível, a uniformização internacional de normas, mas também ao fato de que a Lei Modelo inspirou iniciativas legislativas que se encontram em tramitação no Congresso Nacional.

Aqui, porém, cumpre fazer-se um esclarecimento preliminar. A pesquisa desta dissertação baseou-se no texto no PL n ${ }^{\circ}$ 10.220/18, que resultou do anteprojeto de Lei elaborado pelo Ministério da Fazenda, a partir de texto redigido por professores integrantes do Grupo de Trabalho e previamente debatido nas reuniões do Instituto Brasileiro de Estudos de Recuperação de Empresas - IBR.

Na parte relativa à insolvência transnacional, o PL n ${ }^{\circ} 10.220 / 18$ manteve o texto originalmente proposto. Porém, o PL n ${ }^{\circ} 10.220 / 18$ foi apensado ao Projeto de Lei $\mathrm{n}^{\circ}$ 6.229/05 e resultou em Substitutivo apresentado ao plenário da Câmara dos Deputados em 27 de novembro de 2019, quando estava sendo concluída esta dissertação. Do cotejo dos textos, vê-se que o Projeto de Lei $n^{\circ}$ 6.229/05 incorporou o texto do PL n ${ }^{\circ} 10.220 / 18$, com algumas alterações, que, a nosso sentir, prejudicam a consecução dos propósitos da Lei Modelo.

Este trabalho, no entanto, investiga a hipótese de se incorporar ao ordenamento jurídico brasileiro a Lei Modelo. Para tanto, será feito um estudo dogmático do direito da insolvência transnacional, princípios gerais informadores da matéria, a bem-sucedida experiência norte-americana, como outras fontes relevantes a respeito da matéria, a fim de contribuir, inclusive, com a adequada interpretação e aplicação do direito projetado na matéria, diante da perspectiva de sua promulgação. Além disso, será analisada a compatibilidade do direito projetado sobre insolvências transnacionais com o ordenamento jurídico brasileiro, particularmente com os instrumentos de cooperação internacional positivados e relevantes para o direito empresarial, a saber: (i) a homologação de sentenças estrangeiras, (ii) as cartas rogatórias e (iii) o auxílio direto. 
Muito embora não seja o objeto deste trabalho, a inclusão de breves notas históricas e a contextualização dos temas tratados podem permitir a melhor compreensão da matéria e auxiliar na reflexão, sobretudo quanto aos aspectos de direito comparado relevantes à matéria.

À luz desse estudo inicial, será examinada a Lei Modelo, sua evolução e os principais instrumentos previstos na Lei Modelo voltados à cooperação entre autoridades e coordenação de esforços em nível internacional para contribuir com objetivos comuns no âmbito de um processo de insolvência centralizado na autoridade competente, de acordo com o COMI.

Além disso, será analisado o RIE, sua evolução, algumas semelhanças e diferenças com a Lei Modelo, e as dificuldades interpretativas enfrentadas na aplicação dessas normas por alguns países que adotaram um, outro, ou ambos os sistemas.

Após, passar-se-á para a análise da experiência brasileira no modelo ad hoc a partir de casos concretos, nos quais instrumentos previstos na Lei Modelo foram utilizados para permitir às autoridades brasileiras a proteção ou a busca de bens do devedor no exterior, bem como precedentes que autorizaram o processamento de recuperação judicial de sociedades estrangeiras integrantes de grupos societários com operações no Brasil.

Por fim, esta dissertação pretende contribuir com o desenvolvimento do direito da insolvência transnacional no Brasil, mediante a análise do direito projetado sobre a matéria no PL n $10.220 / 18$, em consonância com as recomendações da Lei Modelo. 


\section{CONCLUSÃO}

A LRE não regula casos de insolvência transnacional. O Código Bustamante, cuja vigência é objeto de debates na doutrina, é ultrapassado. A jurisprudência das cortes superiores sobre reconhecimento de sentenças falimentares estrangeiras é, em geral, pelo não reconhecimento de processos de insolvência estrangeiros. A despeito de não haver recusa expressa quanto à possibilidade de reconhecimento de sentenças estrangeiras de insolvência no Brasil, a jurisprudência tipicamente suscita violação à soberania nacional, exceção de ordem pública à luz de fatos e especificidades para negar o reconhecimento de processos estrangeiros, o que denota um viés territorialista.

A proliferação de recuperações judiciais ajuizadas no brasil por sociedades estrangeiras integrantes de grupos transnacionais evidencia que a LRE, as regras de direito internacional privado e as regras para homologação de sentenças estrangeiras não atendem à realidade atual, sobretudo diante do desenvolvimento de grupos transnacionais e da globalização.

Em matéria de insolvência transnacional, os principais estudiosos da matéria sugerem que os modelos tendentes ao universalismo devem preponderam sobre os modelos territorialistas, o que se reflete também na adoção da Lei Modelo e adesão ao RIE por várias das maiores economias do mundo. Com efeito, sugere-se neste trabalho haver um "movimento trópico" no sentido do universalismo.

O universalismo puro é praticamente inatingível, sobretudo devido à ausência de interesse dos países de renunciarem a sua soberania, interesses nacionais e aos direitos dos credores locais. Com efeito, os Estados soberanos preferem manter o controle sobre procedimentos, regras e políticas aplicáveis em seu território, sobretudo considerando que as regras de insolvência podem ser consideradas como "meta-leis". Por isso, os modelos intermediários, tal como o universalismo modificado, são atualmente mais aceitos.

Em linha com o universalismo modificado, a Lei Modelo estabelece uma recomendação legislativa, que pode ser adotada total ou parcialmente, visando um padrão que permita a aproximação e harmonização dos sistemas jurídicos de diferentes países. Mas, ao mesmo tempo em que a flexibilidade incentiva a adoção por mais países, não 
elimina os principais desafios suscitados pela insolvência transnacional, na medida em que não contém previsões a respeito do possível conflito de normas e os Estados podem criar exceções à sua aplicação, afastando-se do ideal de harmonização.

O Chapter 15 nos EUA retrata os instrumentos previstos na a Lei Modelo para reconhecimento de processos estrangeiros, cooperação e coordenação entre diferentes processos em casos de insolvência transnacional. Os tribunais norte-americanos, em geral, interpretam o Chapter 15 de forma consistente com os objetivos da Lei Modelo, afastando - por exemplo - exceções de política pública nos casos concretos.

A experiência norte-americana permite concluir que seu sucesso se deveu, não apenas à adesão ao texto da Lei Modelo, mas principalmente à interpretação cuidadosa pelas autoridades norte-americanas, coerente com os objetivos postos pela Lei Modelo e com os princípios informadores do sistema.

A ausência de normas a respeito de insolvência transnacional no Brasil não impediu o processamento de processos de recuperação judicial envolvendo sociedades estrangeiras em litisconsórcio com sociedades brasileiras, nem a possibilidade de recorrer a ordenamentos jurídicos estrangeiros para o reconhecimento de processos de insolvência brasileiros. A despeito disso, as soluções dadas pelos tribunais foram limitadas, aplicáveis somente à hipótese de recuperação judicia de veículos utilizados para captação de recursos para financiamento de atividades desenvolvidas no Brasil. Ademais, a complexidade dos casos de insolvência transnacional, bem como a busca por maior segurança jurídica evidenciam a necessidade de disciplinar a matéria, sobretudo considerando que a construção jurisprudencial tende a ser um processo moroso e custoso, que não elimina um elevado nível de incerteza em países de tradição romano-germânica.

Nesse contexto, e considerando a experiência legislativa recente do Chile, foi proposto o PL n ${ }^{\circ} 10.220 / 18$, construído a partir de anteprojeto desenvolvido pelo Grupo de Trabalho e que adota de forma substancial as regras propostas na Lei Modelo.

A criação de norma baseada no universalismo modificado e na Lei Modelo é positiva e capaz de colocar o Brasil em linha com as legislações atuais em matéria de insolvência transnacional. Com efeito, o universalismo modificado (a Lei Modelo e o RIE) são coerentes com o ideal de maximização e preservação de valor, inclusive a partir de 
instrumentos que permitam a superação de crises econômico-financeiras de sociedades viáveis, e com o princípio da paridade entre credores, também prestigiados pelo Brasil.

Ademais, a adoção pelo Brasil de norma inspirada na Lei Modelo não conflitaria com os instrumentos para cooperação jurídica internacional disponíveis no Brasil à luz do artigo $4^{\circ}$, IX da CF e do CPC, notadamente (i) homologação de decisão estrangeira, (ii) carta rogatória e, (iii) auxílio direto.

Contudo, foi recentemente proposto um Substitutivo ao PL $\mathrm{n}^{\circ}$ 6.229/05, que modificou o texto formulado pelo Grupo de Trabalho que originou o PL n ${ }^{\circ} 10.220 / 18$, alterando conceitos-chave da Lei Modelo, o que prejudica consecução dos propósitos da Lei Modelo. Logo, nos parece que seria necessário que o legislador resgatasse, antes da edição da lei, a redação proposta originalmente pelo Grupo de Trabalho em matéria de insolvência transnacional, que originou o PL n ${ }^{\circ}$ 10.220/18.

Ademais, para o sucesso do sistema proposto será imprescindível que o intérprete considere a origem internacional da Lei Modelo, os guias de melhores práticas e a experiência internacional a respeito de insolvência transnacional, o que é ainda mais relevante considerando a tradição brasileira eminentemente territorialista e os julgados das cortes superiores a respeito de reconhecimento de sentenças estrangeiras de insolvência. 


\section{BIBLIOGRAFIA}

AASARU, Epp. The Desirability of 'Centre of Main Interest' as a Mechanism for Allocating Jurisdiction and Applicable Law in Cross-Border Insolvency Law. European Business Law Review, Issue 3, pp. 349 -380, 2011.

ABE, Shin. Japan. In: Ho, Look Chan (Ed.). Cross-Border Insolvency: A Commentary on the UNCITRAL Model Law. London: Globe Law and Business, 2012.

ABRÃO, Nelson. O Novo Direito Falimentar: Nova Disciplina Jurídica da Crise Económica da Empresa. São Paulo: Revista dos Tribunais, 1985.

AYOUB, Luiz Roberto, e, CAVALLI, Cássio. A Construção Jurisprudencial da Recuperação Judicial de Empresas. 2ª Edição. Rio de Janeiro: Forense, 2016.

BAIRD, Douglas G. Elements of Bankruptcy. 4th Ed. New York: Foundation Press, 2006.

BAIRD, Douglas G.; RASMUSSEN, Robert K. The End of Bankruptcy. Chicago Working Papers in Law and Economics. n. 173 (Second Series), December 2002. Disponível em SSRN: <https://ssrn.com/abstract=359241> ou <http://dx.doi.org/10.2139/ssrn.359241>

BECUE, Sabrina Maria Fadel. Insolvência transnacional: As contribuições que Lei Modelo da UNCITRAL pode proporcionar para o Brasil. Tese (Doutorado) Universidade de São Paulo, Faculdade de Direito, 143 folhas, 2018.

BERENDS, André J. The UNCITRAL Model Law on Cross-Border Insolvency: A Comprehensive Overview. Tulane Journal of International and Comparative Law, 1998. 
BERGER, Dora. A Insolvência no Brasil e na Alemanha. Porto Alegre: Sergio Antonio Fabris, 2001.

BEZERRA FILHO, Manoel Justino. Lei de recuperação de empresas e falência: Lei 11.101/2005: comentada artigo por artigo. $7^{\text {a }}$ ed. rev., atual. e ampl., São Paulo: Revista dos Tribunais, 2017.

BLOCK-LIEB, Susan. Turnaround: Reflections on the Present Day Influence of Negotiations on International Bankruptcy at the Fifth Session of the Hague Conference on Private International Law in 1925. Brooklyn Journal of Corporate, Financial \& Commercial Law, Fall 2014. Fordham Law Legal Studies Research Paper N. 2538560. 2014. Disponível em SSRN: 〈https://ssrn.com/abstract=2538560〉.

BORK, Reinhard. Principles of Cross-Border Insolvency Law, Cambridge, Reino Unido: Ed. Intersentia, 2017.

BRASIL. CÂMARA DOS DEPUTADOS. Parecer oferecido em Plenário em 27.11.2019 em relação ao projeto de lei $n^{\circ} 6.229$ de 2005 e respectivos apensos. Disponível em: <https://www.camara.leg.br/proposicoesWeb/prop_mostrarintegra?codteor=1838624>. Acesso em: 30 dez. 2019.

BRASIL. SUPERIOR TRIBUNAL DE JUSTIÇA. Recurso Especial $\mathbf{n}^{\circ} \mathbf{1 . 7 0 5 . 2 2 2 / S P ,}$ Relator Ministro Luis Felipe Salomão, Quarta Turma, julgado em 16 de novembro de 2017.

BRASIL. SUPERIOR TRIBUNAL DE JUSTIÇA. Sentença Estrangeira Contestada n ${ }^{\circ}$ 1.734/PT (2007/0224985-0). Relator Ministro Fernando Gonçalves julgado em 15 de setembro de 2010, publicado em 16 de fevereiro de 2011. 
BRASIL. SUPERIOR TRIBUNAL DE JUSTIÇA. Sentença Estrangeira Contestada ${ }^{\circ}$

1.735/ PT (2007/0140920-4). Relator Ministro Arnaldo Esteves Lima julgado em 12 de maio de 2011, publicado em 3 de junho de 2011.

BRASIL. SUPERIOR TRIBUNAL DE JUSTIÇA. Recurso Especial no 15708-0 - RS 91.0021250-4. Relator Ministro Fernando Gonçalves julgado em 28 de maio de 1996, publicado em 24 de março de 1997.

BRASIL. SUPREMO TRIBUNAL FEDERAL. Sentença Estrangeira no 2.492-6: República Federal da Alemanha (Agravo Regimental). Relator Ministro Xavier de Albuquerque, julgado em 3 de março de 1982, publicado em 2 de abril de 1982.

BRASIL. SUPREMO TRIBUNAL FEDERAL. SE n 2492-6: República Federal da Alemanha (Sentença Estrangeira). Relator Ministro Xavier de Albuquerque, julgado em 28 de abril de 1981, publicado em 14 de maio de 1981.

BRASIL. SUPREMO TRIBUNAL FEDERAL. SE n 993. Revista dos Tribunais, v. 136, v. 136, pp. 824-828, 1942.

BRASIL. SUPREMO TRIBUNAL FEDERAL. SE $\mathbf{n}^{\circ}$ 919. Relator Ministro Plínio Casado. Archivo Judiciário, vol. 30/133, 1933.

BRASIL. TRIBUNAL DE JUSTIÇA DO ESTADO DO RIO DE JANEIRO. Processo n. ${ }^{0}$ 0377620-56.2013.8.19.0001. Vara Empresarial da Comarca da Capital - Rio de Janeiro. Decisão proferida em 21.11.2013.

BRASIL. TRIBUNAL DE JUSTIÇA DO ESTADO DE SÃO PAULO. Processo n. 1030812-77.2015.8.26.0100. $1^{a}$ Vara de Falências e Recuperação Judicial de São Paulo Capital. Decisão proferida em 01.04.2015. 
BRASIL. TRIBUNAL DE JUSTIÇA DO ESTADO DE SÃO PAULO. Processo n. ${ }^{*}$ 1037133-31.2015.8.26.0100. $2^{\text {a }}$ Vara de Falências e Recuperação Judicial de São Paulo Capital., Decisão proferida em 04.05.2015.

BRASIL. TRIBUNAL DE JUSTIÇA DO ESTADO DO RIO DE JANEIRO. Processo n. ${ }^{\circ}$ 0203711-65.2016.8.19.0001. Vara Empresarial da Comarca da Capital - Rio de Janeiro. Decisão proferida em 29.06.2016.

BRASIL. TRIBUNAL DE JUSTIÇA DO ESTADO DE SÃO PAULO. Processo n. ${ }^{\circ}$ 1057756-77.2019.8.26.0100. $2^{\mathrm{a}}$ Vara de Falências e Recuperação Judicial de São Paulo Capital. Decisão proferida em 18.06.2018.

BUFFORD, Samuel. Coordination of Insolvency Cases for International Enterprise Groups: A Proposal, Penn State Law Research Paper No. 1-2014. 2014. Disponível em SSRN: < http://ssrn.com/abstract=2382123>. Acesso em: 5 jan. 2020.

. International Insolvency Law \& International Arbitration - A Preliminary

Perspective. American Bankruptcy Law Journal, 2014. Disponível em SSRN: <http://ssrn.com/abstract=2568682>. Acesso em: 30 dez. 2019.

BUXBAUM, Hannah L., Rethinking International Insolvency: The Neglected Role of Choice-of-Law Rules and Theory. Stanford Journal of International Law, Vol. 36, No. 23, 2000. Disponível em SSRN: < https://ssrn.com/abstract=626461 >. Acesso em: $30 \mathrm{dez}$. 2019.

CAMPANA FILHO, Paulo Fernando. A recuperação judicial de grupos societários multinacionais: contribuições para o desenvolvimento de um sistema jurídico brasileiro a partir do direito comparado - Tese de Doutorado em Direito. São Paulo: Universidade de São Paulo, 2013. 
The Legal Framework for Cross-Border lnsolvency in Brazil. Houston Journal of International Law, v. 32, n. 97-151, 2010.

CARRUTHERS, Bruce G. And HALLIDAY, Terrence C. Rescuing Business. The Making of Corporate Bankruptcy Law in England and the United States. Oxford University Press Inc., New York. Reprinted 2003

CEREZETTI Sheila C. Neder. A recuperação judicial da sociedade por ações, Ed. Malheiros, São Paulo, 2012.

CHAIKA, Ioanna. Insolvency of Group of Companies through the prism of the Recast Insolvency Regulation (EU) 2015/848, 2015. Disponível em SSRN: <https://ssrn.com/abstract=2687623>. Acesso em: $30 \mathrm{dez} .2019$.

CHANDRA MOHAN, S. Cross-border Insolvency Problems: Is the UNCITRAL Model Law the Answer?. International Insolvency Review, pp. 199-223, 2012.

CHONG, Adeline. Recognition of Foreign Judgments and Cross-Border Insolvencies, Singapore Management University School of Law Research Paper No. 8/2014. Disponível em SSRN: 〈http://ssrn.com/abstract=2381950〉. Acesso em: 30 dez. 2019.

CHUNG, John J. In Re Qimonda AG: The Conflict between Comity and the Public Policy Exception in Chapter 15 of the Bankruptcy Code, in: 32 B.U. Int'l L.J. 89, 2014. Disponível em SSRN: 〈http://ssrn.com/abstract=524668>. Acesso em: 30 dez. 2019.

CROCCO, Fabio Weinberg. When deference makes a difference: the role of U.S. Courts in Cross-Border Bankruptcies. III Prize in International Insolvency Studies, 2019 Disponível em: < https://www.iiiglobal.org/sites/default/files/media/Fabio\%20Weinberg\%20Crocco\%20 -\%20When\%20Deference\%20Makes\%20a\%20Difference.pdf $>$. Acesso em: 15 out. 2019. 
DEZEM, Renata Mota Maciel Madeira. A extensão da competência do juízo da recuperação judicial. Tese (Doutorado) - Universidade de São Paulo, Faculdade de Direito, 400 folhas, 2016

DOLINGER, Jacob. Brazilian International Procedural Law. In DOLINGER, Jacob. ROSENN, Keith S. A panorama of Brazilian Law. Rio de Janeiro/Miami, Editora Esplanada/University of Miami North-South Center, 1992. p. 360).

DWORKIN, Ronald M. The Model of Rules, 1967-1968 Chi. L Rev. 14-46, 1967. Disponível em Yale Law School Legal Scholarship Repository: http://users.umiacs.umd.edu/ horty/courses/readings/dworkin-1967-model-of-rules.pdf Acesso em: 10 out. 2019.

EIDENMÜLLER, Horst. A New Framework for Busines Restructuring in Europe: The EU Commission's Proposals for a Reform of the European Insolvency Regulation and Beyond. ECGI - Law Working Paper No. 199/2013, 2013. Disponível em SSRN: <http://ssrn.com/abstract=2230690>. Acesso em: 30 dez. 2019.

Contracting for a European Insolvency Regime, January 2017, Disponível em SSRN: 〈 https://papers.ssrn.com/sol3/papers.cfm?abstract id=2896340> Acesso em: 30 dez. 2019.

EUROPEAN LAW INSTITUTE. Rescue of Business in Insolvency Law. Vienna, 2017 Disponível em <http://www.europeanlawinstitute.eu/fileadmin/user_upload/p_eli/Publications/Instrume nt INSOLVENCY.pdf>. Acesso em: 15 jan. 2020.

EUROPEAN UNION - THE COUNCIL. Report on the Convention of Insolvency Proceedings n. $\quad$ 6500/96. Disponível em: <https://www.r3.org.uk/media/documents/technical_library/Legislation/Virgos-SchmitReport.pdf>. Acesso em: 30 dez. 2019. 
FELSBERG, Thomas Benes, and Paulo Fernando CAMPANA FILHO, Corporate Bankruptcy and Reorganization in Brazil: National and Cross-Border Perspectives, Norton Annual Review of International Insolvency pp. 275-300, 2009,.

FIDDER, Mark Roelof. Conflict of interest involving liquidators. Recommendations to the European Union legislator. Leiden University. Faculty of Law. Thesis for the Master of Laws in Company Law. Disponível em: <https://www.njb.nl/Uploads/2014/9/Thesis---Conflict-of-interest-involving-liquidators--mrfidder.pdf >. Acesso em: 30 dez. 2019.

FISHER, Richard; AL-ATTAR, Adam. The UNCITRAL Model Law. In SHELDON, Richard (Ed.). Cross Border Insolvency. 4. Ed Haywards Heath: Ed. Blomsbury Professional, pp. 112-153, 2015.

FLETCHER, Ian F. Insolvency in Private International Law. Supplement to Second Edition, Oxford University Press, 2007.

Spreading the Gospel: The Mission of Insolvency Law, and the Insolvency Practitioner, in the early Twenty-First Century. In Rebecca Parry and Paul Omar (eds.), International Insolvency Law: Future Perspectives. INSOL Europe, Nottingham Paris 2016, pp. 179-199. Disponível em: < https://core.ac.uk/download/pdf/42392670.pdf>. Acesso em: 10 jan. 2020.

. The Treatment of Set-off under EC Regulation in Insolvency Proceedings. Tijdschrift voor Insolventienrecht (Tvl). 2002/Special Insolventieverordening, n. 147, 2002.

FLETCHER Ian F. e Bob WESSELS. The Long March towards Attaining a Global Approach to Cross-Border Insolvency Cases in a Globalizing World. Law Review of Corporate Reorganisation \& Restructuring 2014-4, pp. 254-282, 2014. 
GHIO, Emilie. European Insolvency Law: Development, Harmonisation, Application and Reform, 2014. Disponível em SSRN: < http://ssrn.com/abstract=2520637>. Acesso em: 10 jan. 2020.

GUERREIRO, José Alexandre Tavares. Sentença estrangeira e "lex mercatoria". Horizontes e fronteiras do comércio internacional. São Paulo. Saraiva, 1994.

HALLOCK, Jesse. Time Out: The Problematic Temporality of COMI Analysis in Chapter 15 Bankruptcy Cases in the Second Circuit, 2015 Columbia Business Law Review. Disponível em SSRN: 〈http://ssrn.com/abstract=2591402〉. Acesso em: 10 jan. 2020.

HENRICI, Ricardo Loretti e CARPENTER, Marcelo Lamego. Recuperação Judicial no Brasil de Empresas Estrangeiras. In: Elias. Luiz Vasco (coord). 10 Anos da Lei de Recuperação de Empresas e Falências - Reflexões Sobre A Reestruturação Empresarial. São Paulo: Quartier Latin, pp. 411-430, 2015.

HO, Look Chan. The Revised UNCITRAL Model Law Enactment Guide - A Welcome Product? Journal of International Banking Law and Regulation, 325, 2014. Disponível em SSRN: <https://ssrn.com/abstract=2434780>. Acesso em: 10 jan. 2020.

INSOL EUROPE. European Communication and Cooperation Guidelines for Crossborder Insolvency. Wessels, Bob; Virgós, Miguel. 2007. Disponível em: <https://www.insol-europe.org/download/documents/1113>. Acesso em: 23 nov. 2019.

INSOL INTERNATIONAL. Insol International Special Report - Chile's New Insolvency Law. Pedro A. Jimenez, Jones Day, USA; Rodolfo Pittaluga Jr., Deloitte Financial Advisory Services LLP, USA, and Pablo Herrera, Deloitte. Disponível em: <https://www.insol.org/_files/TechnicalSeries/Special\%20Reports/Special\%20Report\% 20on\%20Chile\%20-\%2026\%20September\%202014.pdf>. Acesso em: 10 jan. 2019. 
KIPNIS, Alexander M. Beyond Uncitral: Alternatives to Universality in Transnational Insolvency. Denver Journal of International Law and Policy. n. 36. 2008, p. 30-31.

LATELLA, Dario. The 'COMI' concept in the Revision of the European Insolvency Regulation, 2013. Disponível em SSRN: 〈http://ssrn/com/abstract=2336470 $>$. Acesso em: 30 dez. 2019.

LISBOA, Marcos de Barros, A racionalidade econômica da nova lei de falências e de recuperação de empresas. In: Paiva. Luiz Fernando Valente de (Ed.). Direito Falimentar e a Nova Lei de Falências e Recuperação de Empresas. São Paulo: Quartier Latin, 2005.

LoPUCKI, Lynn M. Cooperation in International Bankruptcy: A Post-Universalist Approach. Cornell Law Review, Vol. 84, 696, 701, 1999. Disponível em: <http://ssrn.com/abstract=132408>. Acesso em: $30 \mathrm{dez} .2019$.

. The Case for Cooperative Territoriality in International Bankruptcy.

Michigan Law Review, Vol. 98, 2216, 2000.

Universalism Unravels. American Bankruptcy Law Journal, Vol. 79, 143, (2005). Disponível em: 〈http://ssrn.com/abstract=732123〉. Acesso em: $30 \mathrm{dez}$. 2019.

MARQUES, Claudia Lima; JACQUES, Daniela Corrêa. Normas de aplicação imediata como um método para o Direito Internacional Privado de proteção do consumidor no Brasil. In Cadernos do Programa de Pós-Graduação em Direito da UFRGS, n. 1, 2004, pp.65-96.

MCCORMACK, Gerard, Groups of Companies and the 'Recast' European Insolvency Regulation. In Rebecca Parry and Paul Omar (eds.), Reimagining Rescue, INSOL Europe, Nottingham Paris 2016, pp. 109- 118. Disponível em: 
<http://irep.ntu.ac.uk/id/eprint/28399/1/PubSub5896 Omar.pdf $>$. Acesso em: $30 \mathrm{dez}$. 2019.

MEVORACH, Irit. Appropriate treatment of corporate groups in insolvency: a universal view. European Business Organization Law Review, 2007. Disponível em: 〈http://eprints.nottingham.ac.uk/1772/1/Mevorach_EBOR_2007.pdf $>$. Acesso em: 30 dez. 2019.

The Future of Cross-Border Insolvency Overcoming Biases and

Closing Gaps. Oxford University Press, Oxford, Reino Unido, 2018 (ebook).

MOSS. Gabriel. New World and Old World: Symphony or Cacophony? In Rebecca Parry and Paul Omar (eds.), International Insolvency Law: Future Perspectives. INSOL Europe, Nottingham Paris 2016, pp. 17-43. Disponível em: < https://core.ac.uk/download/pdf/42392670.pdf>. Acesso em: 30 dez. 2019.

NADELMANN, Kurt H. Bankruptcy: Fringe Benefits from Recognition of Foreign Judgements Conventions. Netherlands International Law Review (NILR) 1983.

PISTOR, Katharina. The Standardization of Law and Its Effect on Developing Economies, in: 50 American Journal of Comparative Law 2002, 97. Disponível em: 〈https://unctad.org/en/docs/pogdsmdpbg24d4.en.pdf>. Acesso em: 30 dez. 2019.

PONTES DE MIRANDA, Francisco Cavalcanti. Comentários ao Código de Processo Civil. t. II. 3 ed. atual. por Sérgio Bermudes. Rio de Janeiro: Forense, 1997

POTTOW, John A. E. Beyond Carve-Outs and Toward Reliance: A Normative Framework for Cross-Border Insolvency Choice of Law, 9 Brook. J. Corp. Fin. \& Com. L. (2014). Disponível em: 〈https://brooklynworks.brooklaw.edu/bjcfcl/vol9/iss1/10>. 
Greed and Pride in International Bankruptcy: The Problems and

Proposed Solutions To 'Local Interests.' Paper presented at Academics' Meeting Insol International, Sydney, 12/13 March 2005. Disponível em SSRN: <https://papers.ssrn.com/sol3/papers.cfm?abstract_id=711125>. Acesso em: $30 \mathrm{dez}$. 2019.

Procedural Incrementalism: A Model for International Bankruptcy, Virginia Journal on International Law. 45, no. 4 (2005), Charlottesville: 935-1015. p. 988 e 992.

RAMOS, André de Carvalho. Curso de Direito Internacional Privado - São Paulo: Saraiva Educação, 2018.

RASMUSSEN, Robert K. Where are All the Transnational Bankruptcies?: The Puzzling Case for Universalism. Vanderbilt Law and Economics. Working Paper No. 07-13, 2017. Disponível em: <http://ssrn.com/abstract=982678>. Acesso em: $30 \mathrm{dez}$. 2019.

RECHSTEINER, Beat Walter. Direito Falimentar Internacional e Mercosul. São Paulo: Juarez de Oliveira. 2000.

RECHSTEINER, Beat Walter. Homologação de Sentenças Estrangeiras no Brasil: Breves Considerações. Revista Direito e Desenvolvimento, v. 3, n. 5, janeiro/junho 2012.

RECHSTEINER, Beat Walter. A Insolvência Internacional Sob a Perspectiva do Direito Brasileiro. In: PAIVA. Luiz Fernando Valente de (Ed.). Direito Falimentar e a Nova Lei de Falências e Recuperação de Empresas. São Paulo: Quartier Latin, 2005.

SANTOS, J. A. Penalva. A Falência no Direito Internacional e o Mercosul. In: Santos, Paulo Penalva (Ed.). A Nova Lei de Falências e de Recuperação de Empresas: Lei n ${ }^{\circ}$ 11.101/05. Rio de Janeiro: Forense, 2007. 
SATIRO, Francisco e CAMPANA FILHO, Paulo Fernando. A Insolvência Transnacional: Para Além da Regulação Estatal e na Direção dos Acordos de Cooperação. In: Toledo, Paulo Fernando Campos Salles de; e Satiro, Francisco (Ed.). Direito das Empresas em Crise: Problemas e Soluções. São Paulo: Quartier Latin, 2012, pp. 121-140.

SATIRO, Francisco, e, MORAES PITOMBO, Antônio Sergio A. de. Comentários à Lei de Recuperação de Empresas e Falência. $2^{a}$ ed., ver., atual., e ampl., São Paulo: Revista dos Tribunais, 2007.

SATIRO, Francisco, CAMPANA FILHO, Paulo Fernando; BECUE, Sabrina. Insolvência Transnacional e o Projeto de Código Comercial: o que diz a UNCITRAL sobre credores nacionais e estrangeiros. São Paulo. Jota, 05/07/2016. Disponível em: < https://www.jota.info/paywall?redirect_to=//www.jota.info/opiniao-eanalise/artigos/insolvencia-transnacional-e-o-projeto-de-codigo-comercial-05072016> . Acesso em: 30 dez. 2019.

SEIFE, Howard; VAZQUEZ, Francisco. Experiência Transnacional de Empresas Brasileiras nos Estados Unidos e os Benefícios da Lei Modelo. In: Elias. Luiz Vasco (coord). 10 Anos da Lei de Recuperação de Empresas e Falências - Reflexões Sobre A Reestruturação Empresarial. São Paulo: Quartier Latin, pp. 173-198, 2015.

SEKOLEC, Jernej. UNCITRAL Model Law in Cross-Border Insolvency: An indispensable complement to the EU Insolvency Regulation. Tijdschrift voor Insolventienrecht (Tvl). 2002/Special - Insolventieverordening, n. 147, 2002

SHELDON. Richard QC. Historical Introduction. In SHELDON, Richard (Ed.). Cross Border Insolvency. 4. Ed. Haywards Heath: Ed. Blomsbury Professional, pp.1-13, 2015. 
SOUZA JUNIOR, Francisco Satiro de; e Pitombo, Antonio Sérgio A. de Moraes (org.). Comentários à Lei de Recuperação de Empresas e Falência: Lei 11.101/2005: Artigo por Artigo. $2^{\mathrm{a}}$ ed. São Paulo: RT, 2007.

TELLECHEA, Rodrigo; SCALZILI, João Pedro; SPINELLI, Luis Felipe. História do direito falimentar: da execução pessoal à preservação da empresa. São Paulo: Almedina, 2018.

TELLECHEA, Rodrigo; SCALZILI, João Pedro; SPINELLI, Luis Felipe. Recuperação de Empresas e Falência. Teoria e Prática na Lei 11.101/2005. 3. ed. rev., e ampl. São Paulo: Almedina, 2018.

TEO, Chindar. The cross-border insolvency of international banks (tese de doutorado) - Victoria University, School of Law, Faculty of Business and Law (2013). Disponível em: <http://vuir.vu.edu.au/26289/>. Acesso em: 30 dez. 2019.

THE AMERICAN LAW INSTITUTE. Guidelines Applicable to Court-to-Court Communications in Cross-Border Cases. Philadelphia: The American Law Institute; Toronto: The International Insolvency Institute, 2003. Disponível em: $<$ https://www.bccourts.ca/supreme_court/practice_and_procedure/practice_directions_a nd_notices/General/Guidelines\%20Cross-Border\%20Cases.pdf $>$. Acesso em: $30 \mathrm{dez}$. 2019.

THE AMERICAN LAW INSTITUTE. Transnational Insolvency: Global Principles for cooperation in International Insolvency Cases. Philadelphia: The American Law Institute; Toronto: The International Insolvency Institute, 2012. Disponível em: <https://www.iiiglobal.org/sites/default/files/alireportmarch_0.pdf>. Acesso em: $30 \mathrm{dez}$. 2019.

TOLEDO, Paulo F. C. Salles de. A reforma da lei de falências e a experiência do direito estrangeiro. Revista do Advogado, São Paulo, n.36, p. 82-87, 1992. 
In: Toledo, Paulo F. C. Salles de; Abrão, Carlos Henrique (coords.).

Comentários à Lei de Recuperação de Empresas e Falência. 6. ed. rev. atual. São Paulo: Saraiva, 2016, e-book.

TRI LEIDEN AND NOTTINGHAM LAW SCHOOL. EU Cross-Border Insolvency Court-to-Court Cooperation Principles. Fev. 2015. Disponível em: <http://www.ejtn.eu/PageFiles/16467/EU_Cross-Border_Insolvency_Court-toCourt_Cooperation_Principles.pdf>. Acesso em: 30 dez. 2019.

THEODORO JÚNIOR, Humberto. Curso de Direito Processual Civil - vol. III 48 ed. rev., atual. e ampl. - Rio de Janeiro: Forense, 2016.

THE WORLD BANK. Principles for Effective Insolvency and Creditor/Debtor Regimes. Washington DC, Revised 2015. Disponível em http://www.worldbank.org/en/topic/financialsector/brief/the-world-bank-principles-foreffective-insolvency-and-creditor-rights. Acesso em: 30 dez. 2019.

TORRES PEREIRA, Marcos Vinícius. Falência E Conflito De Jurisdições No Direito Internacional Privado Brasileiro. Encontro Nacional do CONPEDI (Uberlândia, MG) Anais do XXI Encontro Nacional do CONPEDI. - Florianópolis : Fundação Boiteux, 2012. Disponível em: <http://www.publicadireito.com.br/artigos/?cod=794288f252f45d35>.

TUNG, Frederick, Fear of Commitment in International Bankruptcy. George Washington International Law Review, Vol. 33, 2001. Disponível em SSRN: <https://ssrn.com/abstract=277394 or http://dx.doi.org/10.2139/ssrn.277394>. Acesso em: 30 dez. 2019.

Skepticism about Universalism: International Bankruptcy and $\begin{array}{llll}\text { International } & \text { Relations. } & \text { Disponível }\end{array}$ 
https://papers.ssrn.com/sol3/papers.cfm?abstract id=267437\&download=yes ). Acesso em: 30 dez. 2019.

UNITED NATIONS. Legislative Guide on Insolvency Law. UNCITRAL. United Nations: New $2005 . \quad$ York, Disponível em: $<$ http://www.uncitral.org/pdf/english/texts/insolven/05-80722_Ebook.pdf>. Acesso em: em 25 abr. 2018

UNITED NATIONS. UNCITRAL Model Law on Cross-Border Insolvency with Guide to Enactment and Interpretation. New York, 2014. Disponível em: < https://www.uncitral.org/pdf/english/texts/insolven/1997-Model-Law-Insol-2013-GuideEnactment-e.pdf>. Acesso em: 15 jan. 2020.

UNITED NATIONS. UNCITRAL Model Law on Cross-Border Insolvency: The Judicial Perspective. $2013 . \quad$ Disponível em <http://www.uncitral.org/pdf/english/texts/insolven/Judicial-Perspective-2013-e.pdf> . Acesso em 21 jul. 2018.

UNITED NATIONS. UNCITRAL Practice Guide on Cross-Border Insolvency Cooperation. United Nations: New York, 2010. Disponível em: <http://www.uncitral.org/pdf/english/texts/insolven/Practice_Guide_Ebook_eng.pdf >. Acesso em: 25 abr. 2018.

VALVERDE, Trajano de Miranda. Comentários à Lei de Falências: Decreto-lei n. 7.661, de 21 de junho de 1945, vol. I, 4. ed. atual. por J. A. Penalva Santos e Paulo Penalva Santos. Rio de Janeiro: Forense, 2001.

VIO. Daniel de Avila. Grupos Societários - Ensaio sobre os Grupos de Subordinação, de Direito e de Fato, no Direito Societário Brasileiro. São Paulo: Quartier Latin, 2016.

WESSELS, Bob. International Insolvency Law. Part I Global Perspectives on Cross Border Insolvency Law. Volume X, $4^{\text {th }}$ edition. Deventer, Wolters Kluwer, 2015. 
International Insolvency Law. Part II European Insolvency Law. Volume X, $4^{\text {th }}$ edition. Deventer, Wolters Kluwer, 2017.

. On The Future of European Insolvency Law. In Rebecca Parry and Paul Omar (eds.), International Insolvency Law: Future Perspectives. INSOL Europe, Nottingham Paris 2016, pp. 17-43. Disponível em: < https://core.ac.uk/download/pdf/42392670.pdf>. Acesso em: 30 dez. 2019.

.The EU Regulation on Insolvency Proceedings (Recast). Disponível em: <http://bobwessels.nl/site/assets/files/1856/eir-recast-aug-2015-technical-note.pdf>. Acesso em: 25 abr. 2018.

. We Can Work It Out: Cross-border Judicial Cooperation in Insolvency Cases in the EU. Paper apresentado em fevereiro de 2015 durante uma conferência internacional sobre insolvência em Bruxelas. Disponível em: 〈http://bobwessels.nl/wp/wp-content/uploads/2016/08/12.-B.-Wessels.pdf >. Acesso em: 30 dez. 2019.

WESTBROOK, Jay Lawrence. A Global Solution to Multinational Default. Michigan Law Review. n. 98. 2000

Chapter 15 at Last. American Bankruptcy Law Journal, v. 79, 2005.

Transparency in Corporate Groups (November 19, 2018). U of Texas

Law, Public Law Research Paper. Disponível em SSRN: <https://ssrn.com/abstract=3287378>. Acesso em: 30 dez. 2019. 
Chapter 15 Comes of Age (2013). Annual Review of Solvency Law, Janis P. Sarra, ed., Carswell, 2013; U of Texas Law, Public Law Research Paper No. 617. Disponível em SSRN:<https://ssrn.com/abstract=2477878>. Acesso em: 30 dez. 2019.

. Global Insolvency Proceedings for a Global Market: The Universalist System and the Choice of a Central Court (March 28, 2018). U of Texas Law, Public Law Research Paper No. 691. Disponível em

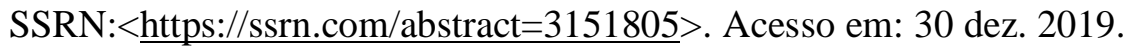

